\title{
A parametric family of quartic Thue inequalities
}

\section{Salah Eddine Rihane ${ }^{1}$, Mohand Ouamar Hernane ${ }^{2}$ and Alain Togbé ${ }^{3}$}

${ }^{1}$ Department of Mathematics and Computer Science

Abdelhafid Boussouf University

Mila 43000, Algeria

e-mail: salahrihane@hotmail.fr

${ }^{2}$ Université des Sciences et de la Technologie Houari-Boumediène (USTHB),

Faculté de Mathématiques, Laboratoire d'Algèbre et Théorie des Nombres

BP 32, 16111 Bab-Ezzouar, Alger, Algérie

e-mail: mhernanedusthb.dz

${ }^{3}$ Department of Mathematics and Statistics

Purdue University Northwest

1401 S, U.S. 421, Westville IN 46391, United States

e-mail: atogbe@pnw.edu

Received: 3 February $2019 \quad$ Revised: 15 June $2021 \quad$ Accepted: 23 September 2021

Abstract: Let $c \neq 0,-1$ be an integer. In this paper, we use the method of Tzanakis to transform the quartic Thue equation $x^{4}-\left(c^{2}+c+4\right) x^{3} y+\left(c^{2}+c+3\right) x^{2} y^{2}+2 x y^{3}-y^{4}=\mu$ into systems of Pell equations. Then, we determine all primitive solutions $(x, y)$ with $0<|\mu| \leq|c+1|$.

Keywords: Parametric Thue equations, Method of Tzanakis, Continued fraction, Linear form in logarithms.

2020 Mathematics Subject Classification: 11D59, 11D09, 11D75, 11D25, 11A55, 11 J86.

\section{Introduction}

In [17], Tzanakis considered Thue equations of the form $F(x, y)=k$, where $F$ is a quartic form that corresponds to a quartic field $\mathbb{K}$, which is the composite of two real quadratic fields. He proved that solving this equation consists in solving a system of Pell equations. Therefore, the 
method is called the Tzanakis method. The method was successfully applied by several authors not only to families of quartic Thue equations but also to families of quartic Thue inequalities. One see for example [2-12,17,19].

It is interesting to notice that for the method one can use the theory of continued fractions to determine small values of $k$ for which the equation $F(x, y)=k$ has a solution. For this, Worley [18], Dujella [1], Dujella-Ibrahimpašić [2], Ibrahimpašić [10] have extended or generalized the classical results of Legendre and Fatou concerning Diophantine approximations of irrational numbers.

In 2007, Leprévost, Pohst, and Schöpp [15] have determined the units of the number field associated with

$$
f_{n, b}(x)=x^{4}-n x^{3}+b(n-1) x^{2}+2 b^{2} x-b^{3},
$$

for $b= \pm 1$. The family of Thue equations associated to this family of polynomials with $n=c^{2}+c-5$ and $b=-1$ was studied in [8]. In this paper, we study the family of Thue equations associated to $f_{n, b}$, with $n=c^{2}+c+4$ and $b=1$.

In fact, let $c \neq-1,0$ be an integer. (Notice that $c=0,-1, f_{n, b}$ is reducible and these cases are not interesting). We consider the following Thue inequality

$$
\left|x^{4}-\left(c^{2}+c+4\right) x^{3} y+\left(c^{2}+c+3\right) x^{2} y^{2}+2 x y^{3}-y^{4}\right| \leq|c+1|
$$

or equivalently the Thue equation

$$
x^{4}-\left(c^{2}+c+4\right) x^{3} y+\left(c^{2}+c+3\right) x^{2} y^{2}+2 x y^{3}-y^{4}=\mu,
$$

where $|\mu| \leq|c+1|$. The aim of this paper is not only to apply again the Tzanakis method to solve inequality (1) but also to add another paper to the already very rich literature. Therefore, we prove the following theorem:

Theorem 1.1. Let $c$ be an integer such that $c \neq 0,-1$. Then, for any $c \neq 1,-2$, the Thue inequality (1) has the primitive solutions $(x, y)= \pm(0,1), \pm(1,0), \pm(1,1)$. The primitive solutions for $c=1,-2$ are $(x, y)= \pm(0,1), \pm(1,-2), \pm(1,0), \pm(1,1), \pm(1,3)$.

We notice that if $c<-1$, then $c^{2}+c+4=(-c-1)^{2}+(-c-1)+4$ and $c^{2}+c+3=$ $(-c-1)^{2}+(-c-1)+3$. Thus, we only need to consider $c \geq 2$ as for $c=1$, we used Maple to determine the solutions cited in Theorem 1.1.

Here is how this paper is organized. We will use the Tzanakis method to transform Thue equation (2) into the following system of Pell equations

$$
V^{2}-\left(c^{2}+4\right) U^{2}=-4 \mu, \quad Z^{2}-\left(c^{2}+\mu 2 c+5\right) U^{2}=-4 \mu .
$$

This is done in the next section. In Section 3, we will recall some important results that will be used in the remaining sections. In Section 4, by means of the results on Diophantine approximations recalled in Section 3 we will obtain some approximations related to the solutions and show that we must consider $\mu= \pm 1, \pm 4$ if $\mu \leq|c+1|$. In Section 5, we use the system of Pell equations obtained in Section 2 to define a linear form in three logarithms of algebraic 
numbers and apply gap principle to bound the parameters. In Section 6, we transform the linear form in three logarithms obtained in Section 5 into a linear form in two logarithms. Then, we can apply a result of Laurent [14]. We combine the lower and upper bounds of this linear form in logarithms to get a contradiction for $c>128073$. This bound of $c$ is low enough to apply a reduction method. This done in Section 7. Finally, we completely prove Theorem 1.1 in the last section.

\section{Application of Tzanakis method}

In this section, we recall the Tzanakis method and use it to transform equation (2) into a system of Pell equations. One can refer to [17] or other references for the details about the method. So let us consider a Thue equation

$$
f(x, y)=\mu,
$$

where

$$
f(x, y)=a_{0} x^{4}+4 a_{1} x^{3} y+6 a_{2} x^{2} y^{2}+4 a_{3} x y^{3}+a_{4} y^{4} \in \mathbb{Z}[x, y], a_{0}>0 .
$$

We assign to this equation the cubic equation

$$
4 \rho^{3}-g_{2} \rho-g_{3}=0
$$

where

$$
g_{2}=a_{0} a_{4}-4 a_{1} a_{3}+3 a_{2}^{2} \in \frac{1}{12} \mathbb{Z}, g_{3}=\left|\begin{array}{lll}
a_{0} & a_{1} & a_{2} \\
a_{1} & a_{2} & a_{3} \\
a_{2} & a_{3} & a_{4}
\end{array}\right| \in \frac{1}{432} \mathbb{Z} .
$$

In 1993, Tzanakis [17] proved that a necessary condition to apply his method is that equation (5) has three rational roots $\rho_{1}, \rho_{2}, \rho_{3}$ that satisfy

$$
\frac{a_{1}^{2}}{a_{0}}-a_{2} \geq \max \left\{\rho_{1}, \rho_{2}, \rho_{3}\right\}
$$

Let $H(x, y)$ and $G(x, y)$ be the quartic and sextic covariants of $f(x, y)$ respectively, i.e.

$$
\begin{gathered}
H(x, y)=-\frac{1}{144}\left|\begin{array}{cc}
\frac{\partial^{2} f}{\partial x^{2}} & \frac{\partial^{2} f}{\partial x \partial y} \\
\frac{\partial^{2} f}{\partial y \partial x} & \frac{\partial^{2} f}{\partial y^{2}}
\end{array}\right| \in \frac{1}{48} \mathbb{Z}[x, y], \\
G(x, y)=-\frac{1}{8}\left|\begin{array}{cc}
\frac{\partial f}{\partial x} & \frac{\partial f}{\partial y} \\
\frac{\partial H}{\partial x} & \frac{\partial H}{\partial y}
\end{array}\right| \in \frac{1}{96} \mathbb{Z}[x, y] .
\end{gathered}
$$

Then, we have $4 H^{3}-g_{2} H f^{2}-g_{3} f^{3}=G^{2}$. Put

$$
H=\frac{1}{48} H_{0}, G=\frac{1}{96} G_{0}, \rho_{i}=\frac{1}{12} r_{i}, i=1,2,3,
$$

then

$$
H_{0}, G_{0} \in \mathbb{Z}[x, y], r_{i} \in \mathbb{Z}, i=1,2,3,
$$

and

$$
\left(H_{0}-4 r_{1} f\right)\left(H_{0}-4 r_{2} f\right)\left(H_{0}-4 r_{3} f\right)=3 G_{0}^{2} .
$$


There exist positive square-free integers $k_{1}, k_{2}, k_{3}$ and quadratic $G_{1}, G_{2}, G_{3} \in \mathbb{Z}[x, y]$ such that

$$
H_{0}-4 r_{i} f=k_{i} G_{i}^{2}, i=1,2,3
$$

and $k_{1} k_{2} k_{3}\left(G_{1} G_{2} G_{3}\right)^{2}=3 G_{0}^{2}$. if $(x, y) \in \mathbb{Z} \times \mathbb{Z}$ is a solution of (3), then we obtain

$$
\begin{aligned}
& k_{2} G_{2}^{2}-k_{1} G_{1}^{2}=4\left(r_{1}-r_{2}\right) \mu, \\
& k_{3} G_{3}^{2}-k_{1} G_{1}^{2}=4\left(r_{1}-r_{3}\right) \mu .
\end{aligned}
$$

Now, we will apply Tzanakis method to equation (2). In this particular case, we have

$$
a_{0}=1, \quad a_{1}=-\frac{c^{2}+c+4}{4}, \quad a_{2}=\frac{c^{2}+c+3}{6}, \quad a_{3}=\frac{1}{2}, \quad a_{4}=-1,
$$

so we get

$$
g_{2}=\frac{c^{4}+2 c^{3}+13 c^{2}+12 c+21}{12}, \quad g_{3}=-\frac{2 c^{6}+6 c^{5}+15 c^{4}+20 c^{3}+45 c^{2}+36 c+162}{432} .
$$

One can see that equation (5) has three rational roots

$$
\rho_{1}=-\frac{2 c^{2}+2 c+9}{12}, \rho_{2}=\frac{c^{2}+4 c+6}{12}, \rho_{3}=\frac{c^{2}-2 c+3}{12} .
$$

Since

$$
\frac{a_{1}^{2}}{a_{0}}-a_{2}=\frac{3 c^{4}+6 c^{3}+19 c^{2}+16 c+24}{48} \geq \max \left\{\rho_{1}, \rho_{2}, \rho_{3}\right\},
$$

so the reduction method can be applied to equation (2). We get

$$
\begin{aligned}
& H_{0}(x, y)-4 r_{1} f(x, y)=3\left(c^{2}+4\right)\left(c^{2}+2 c+5\right) x^{2}(x-2 y)^{2}, \\
& H_{0}(x, y)-4 r_{1} f(x, y)=3\left(c^{2}+2 c+5\right)\left(c x^{2}+2 x y-2 y^{2}\right)^{2}, \\
& H_{0}(x, y)-4 r_{1} f(x, y)=3\left(c^{2}+4\right)\left((c+1) x^{2}-2 x y+2 y^{2}\right)^{2} .
\end{aligned}
$$

Taking

$$
k_{1}=3\left(c^{2}+4\right)\left(c^{2}+2 c+5\right), k_{2}=3\left(c^{2}+2 c+5\right), k_{3}=3\left(c^{2}+4\right),
$$

and

$$
G_{1}=x^{2}-2 x y, G_{2}=c x^{2}+2 x y-2 y^{2}, G_{3}=(c+1) x^{2}-2 x y+2 y^{2},
$$

then, by the method of Tzanakis, solving equation (2) reduces into solving the system of Pell equations

$$
\begin{aligned}
V^{2}-\left(c^{2}+4\right) U^{2} & =-4 \mu \\
Z^{2}-\left(c^{2}+2 c+5\right) U^{2} & =-4 \mu,
\end{aligned}
$$

where

$$
U=x^{2}-2 x y, V=c x^{2}+2 x y-2 y^{2}, Z=(c+1) x^{2}-2 x y+2 y^{2} .
$$




\section{Preliminaries}

In this section, we will recall some useful results for the remaining sections.

Let $p_{k} / q_{k}$ denote the $k$-th convergent of $\alpha$. The following result of Worley [18] and Dujella [1] extends classical results of Legendre and Fatou concerning Diophantine approximations of the form $\left|\alpha-\frac{a}{b}\right|<\frac{1}{2 b^{2}}$ and $\left|\alpha-\frac{a}{b}\right|<\frac{1}{b^{2}}$.

Lemma 3.1 (Worley [18], Dujella [1]). Let $\alpha$ be a real number and a and b coprime nonzero integers, satisfying the inequality

$$
\left|\alpha-\frac{a}{b}\right|<\frac{M}{b^{2}}
$$

where $M$ is a positive real number. Then $(a, b)=\left(r p_{k+1} \pm u p_{k}, r q_{k+1} \pm u q_{k}\right)$, for some $k \geq-1$ and nonnegative integers $r$ and $u$ such that $r u<2 M$.

The explicit version of Lemma 3.1 for $M=2$, was given by Worley ([18, Corollary, p. 206]). Dujella and Ibrahimpašić ([2, Propositions 2.1 and 2.2]) extended Worley's work and gave explicit and sharp versions of Lemma 3.1 for $M=3,4,5, \ldots, 12$. Ibrahimpašić [10] also extended this result to $0 \leq M \leq 13$.

We will apply Lemma 3.1 in order to determine all values of $\mu$ with $|\mu| \leq c$ for which the system of equations (9), (10) has solutions. We need also the following lemma (see [5, Lemma 1]).

Lemma 3.2. Let $\alpha, \beta$ be positive integers such that $\alpha \beta$ is not a prefect square, and let $p_{k} / q_{k}$ denote the $k$-th convergent of continued fraction expansion of $\sqrt{\frac{\alpha}{\beta}}$. Let the sequences $\left(s_{k}\right)$ and $\left(t_{k}\right)$ be defined by (12) for the quadratic irrational $\frac{\sqrt{\alpha \beta}}{\beta}$. Then, for any $r$ and $s$,

$$
\alpha\left(r q_{k+1}+u q_{k}\right)^{2}-\beta\left(r p_{k+1}+u p_{k}\right)^{2}=(-1)^{k}\left(u^{2} t_{k+1}+2 r u s_{k+2}-r^{2} t_{k+2}\right) .
$$

Let us recall the following result due to Laurent [14] on linear forms in two logarithms. For any non-zero algebraic number $\alpha$ of degree $d$ over $\mathbb{Q}$, whose minimal polynomial over $\mathbb{Z}$ is $a \prod_{j=1}^{d}\left(X-\gamma^{(j)}\right)$, we denote by

$$
h(\gamma)=\frac{1}{d}\left(\log |a|+\sum_{j=1}^{d} \log \max \left(1,\left|\gamma^{(j)}\right|\right)\right)
$$

the usual absolute logarithmic height of $\alpha$.

Lemma 3.3. Let $\gamma_{1}>1$ and $\gamma_{2}>1$ be two real multiplicatively independent algebraic numbers, $b_{1}, b_{2} \in \mathbb{Z}$ not both 0 and

$$
\Lambda=b_{2} \log \gamma_{2}-b_{1} \log \gamma_{1}
$$

Let $D:=\left[\mathbb{Q}\left(\gamma_{1}, \gamma_{2}\right): \mathbb{Q}\right]$. Let

$$
h_{i} \geq \max \left\{h\left(\gamma_{i}\right), \frac{\left|\log \gamma_{i}\right|}{D}, \frac{1}{D}\right\} \text { for } i=1,2, \quad b^{\prime} \geq \frac{\left|b_{1}\right|}{D h_{2}}+\frac{\left|b_{2}\right|}{D h_{1}} .
$$

Then, we have

$$
\log |\Lambda| \geq-17.9 \cdot D^{4}\left(\max \left\{\log b^{\prime}+0.38, \frac{30}{D}, 1\right\}\right)^{2} h_{1} h_{2} .
$$

We recall the following result due to Matveev [16]. 
Lemma 3.4. Denote by $\alpha_{1}, \ldots, \alpha_{n}$ algebraic numbers, not 1 or 0 , by $\log \alpha_{1}, \ldots, \log \alpha_{n}$ determinations of their logarithms, by $D$ the degree over $\mathbb{Q}$ of the number field $\mathbb{K}=$ $\mathbb{Q}\left(\alpha_{1}, \ldots, \alpha_{n}\right)$, and by $b_{1}, \ldots, b_{n}$ rational integers. Define $B=\max \left\{\left|b_{1}\right|, \ldots,\left|b_{n}\right|\right\}$ and $A_{i}=$ $\max \left\{D h\left(\alpha_{i}\right),\left|\log \alpha_{i}\right|, 0.16\right\}(1 \leq i \leq n)$, where $h(\alpha)$ denotes the absolute logarithmic Weil height of $\alpha$. Assume that the number

$$
\Lambda=b_{1} \log \alpha_{1}+\cdots+b_{n} \log \alpha_{n}
$$

does not vanish; then

$$
|\Lambda| \geq \exp \left\{-C(n, \chi) D^{2} A_{1} \cdots A_{n} \log (e D) \log (e B)\right\},
$$

where $\chi=1$ if $\mathbb{K} \subset \mathbb{R}$ and $\chi=2$ otherwise and

$$
C(n, \chi)=\min \left\{\frac{1}{\chi}\left(\frac{1}{2} e n\right)^{\chi} 30^{n+3} n^{3.5}, 2^{6 n+20}\right\} .
$$

The following lemma is a slight modification of the original version of Baker-Davenport reduction method. (See [6, Lemma 5a].)

Lemma 3.5. Assume that $M$ is a positive integer. Let $p / q$ be the convergent of the continued fraction expansion of $\delta$ such that $q>6 M$ and let

$$
\eta=\left\|\mu^{\prime} q\right\|-M \cdot\|\delta q\|,
$$

where $\|\cdot\|$ denotes the distance from the nearest integer. If $\eta>0$, then there is no solution of the inequality

$$
0<j \delta-k+\mu^{\prime}<A B^{-j}
$$

in integers $j$ and $k$ with

$$
\frac{\log (A q / \eta)}{\log B} \leq j \leq M
$$

\section{The use of continued fractions}

In this section, we will make a connection between solutions of the system of equations (9), (10) and continued fraction expansion of the corresponding quadratic irrationals. The simple continued fraction expansions of quadratic irrational $\alpha=\frac{a+\sqrt{d}}{b}$ is periodic. This expansion can be obtained using the following algorithm. Multiplying the numerator and the denominator by $b$, if necessary, we may assume that $b \mid\left(d-a^{2}\right)$. Let $s_{0}=a, t_{0}=b$ and

$$
a_{n}=\left[\frac{s_{n}+\sqrt{d}}{t_{n}}\right], \quad s_{n+1}=a_{n} t_{n}-s_{n}, \quad t_{n+1}=\frac{d-s_{n+1}^{2}}{t_{n}}, \quad \text { for } n \geq 0 .
$$

If $\left(s_{j}, t_{j}\right)=\left(s_{k}, t_{k}\right)$ for $j<k$, then

$$
\alpha=\left[a_{0}, \ldots, a_{j-1}, \overline{a_{j}, \ldots, a_{k-1}}\right] .
$$

Applying this algorithm to the quadratic irrational $\sqrt{c^{2}+4}$, we find

$$
\sqrt{c^{2}+4}=\left\{\begin{array}{cl}
{\left[c, \overline{\frac{c}{2}, 2 c}\right]} & \text { if } c \text { is even, } \\
{\left[c, \frac{c-1}{2}, 1,1, \frac{c-1}{2}, 2 c\right]} & \text { if } c \text { is odd. }
\end{array}\right.
$$


For more details, see Tables 1 and 2.

\begin{tabular}{|c|ccccc}
\hline$k$ & 0 & 1 & 2 & 3 & $\cdots$ \\
\hline$s_{k}$ & 0 & $c$ & $c$ & $c$ & $\cdots$ \\
$t_{k}$ & 1 & 4 & 1 & 4 & $\cdots$ \\
$a_{k}$ & $c$ & $\frac{c}{2}$ & $2 c$ & $\frac{c}{2}$ & $\cdots$ \\
\hline
\end{tabular}

Table 1. $c$ even

\begin{tabular}{c|cccccccc}
\hline$k$ & 0 & 1 & 2 & 3 & 4 & 5 & 6 & $\cdots$ \\
\hline$s_{k}$ & 0 & $c$ & $c-2$ & 2 & $c-2$ & $c$ & $c$ & $\cdots$ \\
$t_{k}$ & 1 & 4 & $c$ & $c$ & 4 & 1 & 4 & $\cdots$ \\
$a_{k}$ & $c$ & $\frac{c-1}{2}$ & 1 & 1 & $\frac{c-1}{2}$ & $2 c$ & $\frac{c-1}{2}$ & $\cdots$ \\
\hline
\end{tabular}

Table 2. $c$ odd

Assume now that $(U, V, Z)$ is a nonnegative solution of the system of equations (9), (10). Then $\frac{V}{U}$ is a good rational approximation of $\sqrt{c^{2}+4}$. One can see that

From (9), this implies that

$$
\sqrt{c^{2}+4}+\frac{V}{U}>\sqrt{c^{2}+4}
$$

$$
\begin{aligned}
\left|\sqrt{c^{2}+4}-\frac{V}{U}\right| & =\left|c^{2}+4-\frac{V^{2}}{U^{2}}\right| \cdot\left|\sqrt{c^{2}+4}+\frac{V}{U}\right|^{-1} \\
& <\frac{4|\mu|}{\sqrt{c^{2}+4} \cdot U^{2}} \leq \frac{4(c+1)}{\sqrt{c^{2}+4} \cdot U^{2}}<\frac{5}{U^{2}} .
\end{aligned}
$$

Since the period length of the continued fraction expansion of $\sqrt{c^{2}+4}$ is equal to 2 if $c \geq 2$ is even and 5 if $c \geq 2$ is odd, according to Lemma 3.2, we have to consider only the fractions $\left(r p_{k+1}+u p_{k}\right) /\left(r q_{k+1}+u q_{k}\right)$ for $k=0,1$ if $c \geq 2$ is even and $k=0,1, \ldots, 4$ if $c \geq 2$ is odd, respectively.

By checking all possibilities, it is now easy to prove the following result.

Proposition 4.1. Let $c$ and $\mu$ be integers such that $c \geq 5$ and $0<|\mu| \leq c+1$ and such that equation (9) has a solution in integers $U$ and $V$ with $\operatorname{gcd}(U, V)=1$ or 2. Then

$$
\mu \in\{1,-1,-4,4,-c, c\} \text {. }
$$

Furthermore, all solutions of this equation, where $\operatorname{gcd}(U, V)=1$ or 2 , are given by:

$(U, V)=\left(q_{2 n}, p_{2 n}\right)$ if $\mu=1$ and c even;

$(U, V)=\left(q_{5 n}, p_{5 n}\right),\left(2 q_{5 n+4}, 2 p_{5 n+4}\right)$ if $\mu=1$ and codd;

$(U, V)=\left(2 q_{2 n+1}, 2 p_{2 n+1}\right)$ if $\mu=-1$ and c even;

$(U, V)=\left(q_{5 n+3}, p_{5 n+3}\right)$ if $\mu=-1$ and $c$ odd;

$(U, V)=\left(2 q_{2 n}, 2 p_{2 n}\right)$ if $\mu=4$ and c even;

$(U, V)=\left(2 q_{5 n}, 2 p_{5 n}\right)$ if $\mu=4$ and codd;

$(U, V)=\left(2 q_{5 n+3}, 2 p_{5 n+3}\right)$ if $\mu=-4$ and codd;

$(U, V)=\left(2 q_{2 n+1}+q_{2 n}, 2 p_{2 n+1}+p_{2 n}\right),\left(q_{2 n}-2 q_{2 n-1}, p_{2 n}-2 p_{2 n-1}\right)$ if $\mu=c$ and c even; 


$$
\begin{aligned}
(U, V)= & \left(2 q_{5 n+2}, 2 p_{5 n+2}\right),\left(2 q_{5 n+4}-q_{5 n+3}, 2 p_{5 n+4}-p_{5 n+3}\right),\left(q_{5 n}+2 q_{5 n-1}, 2 p_{5 n}+2 p_{5 n-1}\right) \\
& \text { if } \mu=c \text { and } c \text { odd; } \\
(U, V)= & \left(2 q_{2 n+1}-q_{2 n}, 2 p_{2 n+1}-p_{2 n}\right),\left(q_{2 n}+2 q_{2 n-1}, p_{2 n}+2 p_{2 n-1}\right) \quad \text { if } \mu=-c \text { and c even; } \\
(U, V)= & \left(2 q_{5 n+1}, 2 p_{5 n+1}\right),\left(2 q_{5 n+4}+q_{5 n+3}, p_{5 n+4}+p_{5 n+3}\right),\left(q_{5 n}-2 q_{5 n-1}, p_{5 n}-2 p_{5 n-1}\right) \\
& \text { if } \mu=-c \text { and } c \text { odd. }
\end{aligned}
$$

On the other hand, equation (10) can be rewritten into the form

$$
Z^{2}-\left((c+1)^{2}+4\right) U^{2}=-4 \mu .
$$

One can see that $c+1$ in this equation as $c$ in (9), so we have $\mu= \pm 1, \pm 4, \pm(c+1)$. Therefore, if equations (9) and (10) have a common solution $(U, V, Z)$ in integers, then $\mu= \pm 1, \pm 4$.

\section{Gap principle}

Now, let us consider the system of Pell equations

$$
\begin{aligned}
V^{2}-\left(c^{2}+4\right) U^{2} & = \pm 4, \\
Z^{2}-\left(c^{2}+2 c+5\right) U^{2} & = \pm 4 .
\end{aligned}
$$

By [13, Theorems 3.5 and 3.6], we have

$$
U=\frac{\alpha^{j}+(-1)^{j+1} \alpha^{-j}}{\sqrt{c^{2}+4}}=\frac{\beta^{k}+(-1)^{k+1} \beta^{-k}}{\sqrt{c^{2}+2 c+5}},
$$

for some integers $j \geq 0$ and $k \geq 0$, where $j$ and $k$ are both even or odd integers, $\alpha=\frac{c+\sqrt{c^{2}+4}}{2}$ and $\beta=\frac{c+1+\sqrt{c^{2}+2 c+5}}{2}$ are the fundamental solutions of equations (13) and (14) respectively. Notice that if $j$ and $k$ are both even, then the second member of the system (13) and (14) is equal to 4 , otherwise it is equal to -4 .

Note that $j=k=0$ and $j=k=1$ are solutions of the equation (15). So from now we assume that $j \geq 2$ and $k \geq 2$.

Define

$$
\Lambda=j \log \alpha-k \log \beta+\log \left(\sqrt{\frac{c^{2}+2 c+5}{c^{2}+4}}\right) .
$$

We will prove the next two results.

Lemma 5.1. If equation (15) holds with $j \geq 2$, then $0<|\Lambda| \leq \frac{\alpha^{2}}{\alpha^{2}-1} \cdot \alpha^{-2 j}$.

Proof. It is easy to see that $\alpha^{j}$ and $\beta^{k}>1$, for $j, k \geq 1$. We distinguish to cases according to the parity of $k$ and $j$.

Case 1. $k$ and $j$ are both even. Assuming that $\alpha^{j} \geq \beta^{k}$, then $\alpha^{j}-\alpha^{-j} \geq \beta^{k}-\beta^{-k}$. This and $\sqrt{c^{2}+4}<\sqrt{c^{2}+2 c+5}$ will give us $\frac{\alpha^{j}-\alpha^{-j}}{\sqrt{c^{2}+4}}>\frac{\beta^{k}-\beta^{-k}}{\sqrt{c^{2}+2 c+5}}$. This contradicts (15). So, we deduce that $\alpha^{j}<\beta^{k}$, which implies $\frac{1-\beta^{-2 k}}{1-\alpha^{-2 j}}>1$. Thus, we have

$$
0<|\Lambda|=\log \left(\frac{1-\beta^{-2 k}}{1-\alpha^{-2 j}}\right)<-\log \left(1-\alpha^{-2 j}\right)<\frac{\alpha^{2}}{\alpha^{2}-1} \alpha^{-2 j} .
$$


Case 2. $k$ and $j$ are both odd. Let

$$
P=\frac{\alpha^{j}}{\sqrt{c^{2}+4}}, \quad Q=\frac{\beta^{j}}{\sqrt{c^{2}+2 c+5}} .
$$

Then, we have

$$
P+\frac{P^{-1}}{c^{2}+4}=Q+\frac{Q^{-1}}{c^{2}+2 c+5}
$$

i.e.

$$
P-Q=\frac{Q^{-1}}{c^{2}+2 c+5}-\frac{P^{-1}}{c^{2}+4}=\frac{\left(c^{2}+4\right) P-\left(c^{2}+2 c+5\right) Q}{P Q\left(c^{2}+4\right)\left(c^{2}+2 c+5\right)} .
$$

We deduce that

$P Q\left(c^{2}+4\right)\left(c^{2}+2 c+5\right)(P-Q)=\left(c^{2}+4\right) P-\left(c^{2}+2 c+5\right) Q=\left(c^{2}+4\right)(P-Q)-(2 c+1) Q$.

This means that

$$
\left(P Q\left(c^{2}+2 c+5\right)-1\right)\left(c^{2}+4\right)(P-Q)=-(2 c+1) Q<0
$$

Thus, one can see that $P-Q<0$, i.e.,

$$
\Lambda=\log \left(\frac{1+\beta^{-2 k}}{1+\alpha^{-2 j}}\right)<0 .
$$

Therefore, we get

$$
0<|\Lambda|=\log \left(\frac{1+\alpha^{-2 j}}{1+\beta^{-2 k}}\right)<\alpha^{-2 j}<\frac{\alpha^{2}}{\alpha^{2}-1} \alpha^{-2 j} .
$$

Therefore, we get the desired result.

Lemma 5.2. If equation (15) holds with $j \geq 2$ and $c \geq 2$, then

$$
k>\Delta \cdot \alpha \log \alpha,
$$

where $\Delta=j-k \geq 2$ is an even integer.

Proof. We have seen that $\alpha^{j}<\beta^{k}$. It follows that

$$
\frac{j}{k}<\frac{\log \beta}{\log \alpha} .
$$

On the other hand, let

$$
u_{j}(c)=\frac{\alpha^{j}+(-1)^{j+1} \alpha^{-j}}{\sqrt{c^{2}+4}}, \quad u_{k}(c+1)=\frac{\beta^{k}+(-1)^{k+1} \beta^{-k}}{\sqrt{c^{2}+2 c+5}} .
$$

The function $u_{j}(c)$ is increasing if $j$ or $c$ is fixed. Therefore, the equality $u_{j}(c)=u_{k}(c+1)$ implies that $j>k$. Now if we suppose $\Delta=j-k$, then inequality (17) gives

$$
\begin{aligned}
\frac{\Delta}{k} & <\frac{\log \beta}{\log \alpha}-1=\frac{\log (\beta / \alpha)}{\log \alpha}=\frac{\log (1+(\beta-\alpha) / \alpha)}{\log \alpha}<\frac{\beta-\alpha}{\alpha \log \alpha} \\
& =\frac{c+1+\sqrt{c^{2}+2 c+5}-c-\sqrt{c^{2}+4}}{2 \alpha \log \alpha}=\frac{1+\frac{2 c+1}{\sqrt{c^{2}+2 c+5}+\sqrt{c^{2}+4}}}{2 \alpha \log \alpha} \\
& <\frac{1+\frac{2 c+1}{2 \sqrt{c^{2}+4}}}{2 \alpha \log \alpha}<\frac{1}{\alpha \log \alpha},
\end{aligned}
$$

for $c \geq 2$. Therefore, we have

$$
k>\Delta \cdot \alpha \log \alpha
$$




\section{A linear form in two logarithms}

To apply Lemma 3.3, we rewrite $\Lambda$ given by equation (16) into the form

$$
\Lambda=\log \left(\alpha^{\Delta} \cdot \sqrt{\frac{c^{2}+2 c+5}{c^{2}+4}}\right)-k \log \left(\frac{\beta}{\alpha}\right) .
$$

Put

$$
D=4, \quad b_{1}=k, \quad \gamma_{1}=\frac{\beta}{\alpha}, \quad b_{2}=1, \quad \gamma_{2}=\alpha^{\Delta} \cdot \sqrt{\frac{c^{2}+2 c+5}{c^{2}+4}} .
$$

As $\alpha=\frac{c+\sqrt{c^{2}+4}}{2}$ and $\beta=\frac{c+1+\sqrt{c^{2}+2 c+5}}{2}$, one can verify that $\gamma_{1}$ is a root of

$$
x^{4}+c(c+1) x^{3}-\left(2 c^{2}+2 c+3\right) x^{2}+c(c+1) x+1 .
$$

The absolute values of its conjugates greater than 1 are $\beta / \alpha$ and $-\alpha \beta$. Hence, we have

$$
h\left(\gamma_{1}\right)=\frac{1}{4}\left(\log \left(\frac{\beta}{\alpha}\right)+\log (\alpha \beta)\right)=\frac{1}{2} \log \beta .
$$

Thus, we choose

$$
h_{1}:=\frac{1}{2} \log \beta \geq \max \left\{h\left(\gamma_{1}\right), \frac{\left|\log \gamma_{1}\right|}{4}, \frac{1}{4}\right\} .
$$

Also, it is easy to see that

$$
h\left(\alpha^{\Delta}\right)=\frac{\Delta}{2} \log \alpha<\frac{\Delta}{2} \log \beta
$$

and

$$
h\left(\sqrt{\frac{c^{2}+2 c+5}{c^{2}+4}}\right) \leq \log \left(c^{2}+2 c+5\right)<\log \beta .
$$

Thus, we have

$$
h\left(\gamma_{2}\right) \leq h\left(\alpha^{\Delta}\right)+h\left(\sqrt{\frac{c^{2}+2 c+5}{c^{2}+4}}\right)<\frac{\Delta+2}{2} \log \beta .
$$

Then, we take

$$
h_{2}:=\frac{\Delta+2}{2} \log \beta \geq \max \left\{h\left(\gamma_{2}\right), \frac{\left|\log \gamma_{2}\right|}{4}, \frac{1}{4}\right\} .
$$

As $c \geq 2$, we have $\beta=\frac{c+1+\sqrt{c^{2}+2 c+5}}{2}>3.3$. This implies that $\frac{\left|b_{2}\right|}{D h_{1}}=\frac{1}{2 \log \beta}<0.42$. Therefore, we take

$$
b^{\prime}:=\frac{k}{2(\Delta+2) \log \beta}+0.42 .
$$

We suppose that $c \geq 5000$, then $\alpha=\frac{c+\sqrt{c^{2}+4}}{2}>5000$. From Lemma 5.2, we have

$$
\frac{k}{2(\Delta+2) \log \beta}>\frac{\Delta}{2(\Delta+2)} \cdot \frac{\log \alpha}{\log \beta} \cdot \alpha \geq 0.25 \cdot \frac{\log \alpha}{\log \beta} \cdot \alpha>0.25 \alpha>1250 .
$$

This implies $\log b^{\prime}+0.38 \geq 7.51>30 / D$. Therefore, by Lemma 3.3 we obtain

$$
\log |\Lambda| \geq-1145.6 \cdot\left(\log b^{\prime}+0.38\right)^{2} \cdot(\Delta+2) \cdot \log ^{2} \beta .
$$


On the other hand, from Lemma 5.1 we get that

$$
\log |\Lambda|<\log \left(\frac{\alpha^{2}}{\alpha^{2}-1}\right)-2 j \log \alpha<0.001-1.99 j \log \beta .
$$

Combining (20) and (21), we have

$$
\frac{j}{\log \beta}<\frac{0.001}{1.99 \log ^{2} \beta}+\frac{1145.6}{1.99} \cdot\left(\log b^{\prime}+0.38\right)^{2} \cdot(\Delta+2) .
$$

Since $j>k$, we obtain

$$
\frac{k}{2(\Delta+2) \log \beta}<0.0001+287.84 \cdot\left(\log b^{\prime}+0.38\right)^{2} .
$$

It follows that

$$
b^{\prime}<0.4201+287.84 \cdot\left(\log b^{\prime}+0.38\right)^{2} .
$$

So we have $b^{\prime}<33585.6$. Therefore, we get

$$
k<2 \cdot 33585.4 \cdot(\Delta+2) \cdot \log \beta .
$$

Combining this and $k>\Delta \cdot \alpha \log \alpha$, we obtain

$$
\alpha<2 \cdot 33585.4 \cdot \frac{\Delta+2}{\Delta} \cdot \frac{\log \beta}{\log \alpha} \leq 67170.8 \cdot 2 \cdot 1.001<134476 .
$$

Since $c<\alpha$, this implies $c<134476$.

\section{The use of the reduction method}

In this section, we will use another theorem for the lower bounds of linear forms in logarithms which is different from that in Section 5, and Baker-Davenport reduction method to deal with the remaining cases, i.e., $c<134476$.

Now, we apply Lemma 3.4 with $n=3$ and $\chi=1$ to $\Lambda$ defined by equation (16). We take

$$
D=4, \quad b_{1}=j, \quad b_{2}=-k, \quad b_{3}=1, \quad \alpha_{1}=\alpha, \quad \alpha_{2}=\beta, \quad \alpha_{3}=\sqrt{\frac{c^{2}+2 c+5}{c^{2}+4}} .
$$

From the computations in the above section, we can set

$$
h\left(\alpha_{1}\right)=\frac{1}{2} \log \alpha, \quad h\left(\alpha_{2}\right)=\frac{1}{2} \log \beta, \quad h\left(\alpha_{3}\right)=\frac{1}{2} \log \left(c^{2}+2 c+5\right) .
$$

Therefore, we take

$$
A_{1}=2 \log \alpha, \quad A_{2}=2 \log \beta, \quad A_{3} \leq 4 \log \left(c^{2}+2 c+5\right), \quad B=j .
$$

Using Matveev's result, we have

$$
\log |\Lambda| \geq-\frac{3}{2} e \cdot 30^{6} \cdot 3^{3.5} \cdot 4^{2} \cdot 2 \log \alpha \cdot 2 \log \beta \cdot 4 \log \left(c^{2}+2 c+5\right) \cdot \log (4 e) \cdot \log (e j)
$$

By (21), we have

$$
\log |\Lambda|<0.001-1.99 j \log \alpha
$$


Combining the two bounds for $\log |\Lambda|$, we get

$$
\frac{j}{\log (e j)}<4.25 \cdot 10^{13} \log \left(c^{2}+2 c+5\right) \log \beta .
$$

As $\beta<c+2$ and $c<134476$ the above inequality gives us $j<5 \cdot 10^{17}$.

In order to deal with the remaining cases $2 \leq c \leq 134476$, we will use a Diophantine approximation algorithm called the Baker-Davenport reduction method by applying Lemma 3.5 with

$$
\delta=\frac{\log \alpha}{\log \beta}, \quad \mu^{\prime}=\frac{\log \left(\sqrt{\frac{c^{2}+2 c+5}{c^{2}+4}}\right)}{\log \beta}, \quad A=\frac{\alpha^{2}}{\left(\alpha^{2}-1\right) \log \beta}, \quad B=\alpha^{2}
$$

and $M=5 \cdot 10^{17}$.

The program was developed in PARI/GP running with 200 digits. For the computations, if the first convergent such that $q>6 M$ does not satisfy the condition $\eta>0$, then we use the next convergent until we find one that satisfies the condition. We checked in the range $2 \leq c \leq 134476$. In 15 minutes, all the computations were done. For the first run, we obtained $j \leq 24$. The second run gave us $j \leq 5$. We ran the program for the third time and got $j \leq 3$.

For $0 \leq k \leq j \leq 3$, we compute all $U$. Equation (15) has only the integer solutions $j=k=0$ and $j=k=1$. Therefore, we prove the following result.

Theorem 7.1. Let $c \geq 2$ be an integer. All solutions of the system of Pell equations

$$
\begin{aligned}
V^{2}-\left(c^{2}+4\right) U^{2} & =-4, \\
Z^{2}-\left(c^{2}+2 c+5\right) U^{2} & =-4
\end{aligned}
$$

are given by $(U, V, Z)=( \pm 1, \pm c, \pm(c+1))$ and all solutions of the system of Pell equations

$$
\begin{aligned}
V^{2}-\left(c^{2}+4\right) U^{2} & =4, \\
Z^{2}-\left(c^{2}+2 c+5\right) U^{2} & =4
\end{aligned}
$$

are given by $(U, V, Z)=(0, \pm 2, \pm 2)$.

\section{Proof of Theorem 1.1}

Let $(x, y)$ be solution of the inequality (1), and let $U=x^{2}-2 x y, V=c x^{2}+2 x y-2 y^{2}$, $Z=(c+1) x^{2}-2 x y+2 y^{2}$. Then, $(U, V, Z)$ satisfies the system (9) and (10), for $\mu= \pm 1, \pm 4$.

- If $\mu=1$, then by Theorem 7.1 we have $(U, V, Z)=( \pm 1, \pm c, \pm(c+1))$. Thus, $\pm 1=U=$ $x(x-2 y)$ implies that $x= \pm 1$, and $y=0$ or $y=x$. Therefore, we get $(x, y)=( \pm 1,0)$, $\pm(1,1)$.

- If $\mu=-1$, Theorem 7.1 implies $(U, V, Z)=(0, \pm 2, \pm 2)$. The fact $0=U=x(x-2 y)$ gives $x=0$ or $x=2 y$. The case $x=0$ implies $y= \pm 1$. If $x=2 y$, then equations $\pm 2=V=c x^{2}+2 x y-2 y^{2}=4 c y^{2}+2 y^{2}$ provide a contradiction. Therefore, we only obtain the solutions $(x, y)=(0, \pm 1)$. 
- If $\mu=4$, then system (9) and (10) has the form:

$$
\begin{aligned}
V^{2}-\left(c^{2}+4\right) U^{2} & =-16, \\
Z^{2}-\left(c^{2}+2 c+5\right) U^{2} & =-16 .
\end{aligned}
$$

By Proposition 4.1, for $\mu=4$ one can see that $U, V$ and $Z$ are even. We can use substitute $U_{1}=\frac{U}{2}, V_{1}=\frac{V}{2}, Z_{1}=\frac{Z}{2}$ to the system to obtain

$$
\begin{aligned}
V_{1}^{2}-\left(c^{2}+4\right) U_{1}^{2} & =-4 \\
Z_{1}^{2}-\left(c^{2}+2 c+5\right) U_{1}^{2} & =-4 .
\end{aligned}
$$

From Theorem 7.1, we obtain $U_{1}= \pm 1$. It implies that $\pm 2=U=x(x-2 y)$ and these equations have no integer solution.

- If $\mu=-4$, the system (9) and (10) has the form:

$$
\begin{aligned}
V^{2}-\left(c^{2}+4\right) U^{2} & =16 \\
Z^{2}-\left(c^{2}+2 c+5\right) U^{2} & =16
\end{aligned}
$$

Similarly, we get the system

$$
\begin{aligned}
V_{1}^{2}-\left(c^{2}+4\right) U_{1}^{2} & =4 \\
Z_{1}^{2}-\left(c^{2}+2 c+5\right) U_{1}^{2} & =4 .
\end{aligned}
$$

By Theorem 7.1, we obtain $U_{1}=0$. We deduce that that $0=U=x(x-2 y)$, so $x=0$ or $x=2 y$. The case $x=0$ implies that $y^{4}=4$ and the case $x=2 y$ implies that $\pm 4=V=c x^{2}+2 x y-2 y^{2}=4 c y^{2}+2 y^{2}$ providing a contradiction.

For $c=2,3,4$, we used Maple to determine all solutions of Thue inequality (1). We see that the Thue inequality has the solutions listed in Theorem 1.1.

This completes the proof of Theorem 1.1.

\section{Acknowledgements}

The authors are grateful to the referees for useful comments to improve this paper particularly the statement of Proposition 4.1. They also thank Professor Bo He for fruitful discussion.

\section{References}

[1] Dujella, A. (2004). Continued fractions and RSA with small secret exponent. Tatra Mountains Mathematical Publications, 29, 101-112.

[2] Dujella, A., \& Ibrahimpašić, B. (2008). On Worley's theorem in Diophantine approximations. Annales Mathematicae et Informaticae, 35, 61-73. 
[3] Dujella, A., Ibrahimpašić, B., \& Jadrijević, B. (2011). Solving a family of quartic Thue inequalities using continued fractions. Rocky Mountain Journal of Mathematics, 41(4), $1173-1182$.

[4] Dujella, A., \& Jadrijević, B. (2002). A parametric family of quartic Thue equations. Acta Arithmetica, 101(2), 159-170.

[5] Dujella, A., \& Jadrijević, B. (2004). A family of quartic Thue inequalities. Acta Arithmetica, 111(1), 61-76.

[6] Dujella, A., \& Pethő, A. (1998). A generalization of a theorem of Baker and Davenport, The Quarterly Journal of Mathematics Oxford Series (2), 49(3), 291-306.

[7] He, B., Jadrijević, B. \& Togbé, A. (2009) Solutions of a class of quartic Thue inequalities. Glasnik Matematicki Series III, 44, 309-321.

[8] He, B., Kihel, O., \& Togbé, A. (2011). Solutions of a class of quartic Thue inequalities. Computers \& Mathematics with Applications, 61, 2914-2923.

[9] Jadrijević, B. (2011). A parametric family of quartic Thue inequalities. Bulletin of the Malaysian Mathematical Sciences Society, 34(2), 215-230.

[10] Ibrahimpašić, B. (2013). Explicit version of Worley's theorem in Diophantine approximations. Bulletin of International Mathematical Virtual Institute, 3, 59-68.

[11] Jadrijević, B. (2005). A system of Pellian equations and related two-parametric family of quartic Thue equations. Rocky Mountain Journal of Mathematics, 35(2), 547-571.

[12] Jadrijević, B. (2005). On two-parametric family of quartic Thue equations. Journal de Théorie des Nombres de Bordeaux, 17, 161-167.

[13] Keskin, R., \& Duman, M. G. (2019). Positive Integer Solutions of Some Pell Equations. Palestine Journal of Mathematics, 8(2), 213-226.

[14] Laurent, M. (2008). Linear forms in two logarithms and interpolation determinants II. Acta Arithmetica, 133(4), 325-348.

[15] Leprévost, F., Pohst, M., \& Schöpp, A. (2007). Units in some parametric families of quartic fields. Acta Arithmetica, 127(3), 205-216.

[16] Matveev, E. M. (2000). An explicit lower bound for a homogeneous rational linear form in logarithms of algebraic numbers II. Izvestiya: Mathematics, 64(6), 1217-1269.

[17] Tzanakis, N. (1993). Explicit solution of a class of quartic Thue Equations. Acta Arithmetica, 64(3), 271-283.

[18] Worley, R. T. (1981). Estimating $|\alpha-p / q|$. Journal of the Australian Mathematical Society, 31(2), 202-206.

[19] Ziegler, V. (2006). On certain family of quartic equations with three parameters. Glasnik Matematicki Series III, 41(1), 9-30. 